\begin{tabular}{|r|r|r|r|r|r|r|r|r|r|}
\hline & $a$ & $b$ & $c$ & $d$ & $e$ & $f$ & $g$ & $h$ & $i$ \\
\hline $\begin{array}{c}\text { Amount of } \\
\text { blue }\end{array}$ & 0 & $33^{*}$ & $33^{*}$ & $31^{*}$ & $42^{*}$ & 60 & $64^{*}$ & $120^{*}$ & $270^{*}$ \\
$\begin{array}{c}\text { Total excita- } \\
\text { tion of green } \\
\text { Amount of } \\
\text { green }\end{array}$ & $>4000$ & 94 & 49 & 41 & $35 \cdot 5$ & 35 & 34 & 35 & 35 \\
\hline
\end{tabular}

accompanying table in each column the combinations of green and blue are given which make a spot $P$ vanish (wave-length $480 \mathrm{~m} \mu$ ). The amounts marked with an asterisk have been adjusted by the observer. As the blue fleld excites the green receptors also, the amounts of green have been corrected for this excitation (row 2). The sudden increase of the excitation of green from $e$ to $d$, and the increase of the amount of blue from $d$ to $e$, etc., shows that the interaction between
the different mechanisms is small.

The visual acuity of the blue receptors is very small; on a green fleld $P$ is seen as a blue gloom without a deflnite shape.

(b) The same results were found by a second method. Here two semicircular flelds of the colour-mixing apparatus were used; the limits were determined where a difference between the upper and lower fleld became visible. Blue light, green light and mixtures of was varied.

These experiments are equivalent to measurements of wave-length discrimination, and hence wave-length discrimination can be explained by the theory used above, namely, that the eye includes three separate mechanismos, each having its own intensity discrimination and visual acuity.

Analogous measurements were carried out on the green mechanism. A green spot was used $(\lambda=550 \mathrm{~m} \mu)$. The large fleld was illuminated by green and/or red light. No deflnite results were obtained for three observers, $G, P$ and $L$, but we are sure that the intensity discrimination of the green mechanism is nearly as good as for the red mechanism. According to the measurements already reported ${ }^{1}, G$ had an exceptionally large number of green receptors indeed his diserimination of $P$ on a red field was 20 per cent better than for $P$ or $L$. The green of $P$ on a red field was 20 per cent better than for $P$ or $L$. The green mechanism of $B$, however, showed a reduction of the discrimination by a factor of two. Indeed, she had a very low number of green receptors since her luminosity curve was only 4 per cent above my own curve (see ref. 1). Her reading of Stilling's test cards was somewhat reduced. In the Rayleigh test she required an amount of red that was $Q$ times as large as the amount used by a 'normal' observer ; $Q$ being especially since her luminosity curve is very low. Moreover, for $G$, $Q$ was $1 \cdot 15$, but $G$ read the test cards very well.

$Q$ was 115 , but $G$ read the test cards very well. indeed he read the test cards very badly; even worse than $K$ indeed he read the test cards very badly; even worse than $\frac{K}{2}$ (deuteranomalous) $K$ ' The coefficient $Q$ for $D$ and $K$ was $0 \cdot 9$ and $0 \cdot 3$ mine and therefore he had more green receptors and a better colour

We finally conclude that a flicker photometer may be a valuable instrument in the detection of colour blindness (but combined with the anomaloscope), especially because the colour-blind can adjust the minimum of flicker very accurately. Settings which suggest unusual sensitiveness or insensitiveness to red point to a colour-discrimination.

These measurements will be reported in more detail elsewhere. Our thanks are due to Prof. G. F. Rochat for his help and advice in the clinical tests.

Natuurkundig Laboratorium de

Rijks-Universiteit, Groningen. March 25.

de Vries, Hl., Nature, 157, 736 (1946)

${ }^{2}$ de Vries, Hl., J. Opt. Soc. Amer., 36 (Feb. 1946)

\section{Foveal Colour Sensitivity}

RECENTLX, during the measurement of the hue discrimination curves for small areas of the retina within the central fovea, it was noticed that if the two halves of the small circular matching field (diameter subtending $15^{\prime}$ at the eye) were set at the same wavelength $\lambda$, the lower beam, which did not quite match the upper beam in colour, had to be displaced slightly to a new wave-length $\lambda+\lambda$ in colour, had to be displaced slightly to a new

These differences $(\Delta \lambda)$ for various wave-lengths are shown in the accompanying graph. The full curve was obtained when the matching fleld was viewed directly so that its image lay upon the retina at th flxation point; whereas the broken curve was found when the fleld was displaced $20^{\prime}$ into the left visual field by the use of a small fixation light. Between $0.560 \mu$ and $0.600 \mu$ the curves appear to be the same, and throughout most of the spectrum investigated when both flelds were set to the same wave-length, the lower fleld appeared slightly more blue than the upper one. Beyond $0.510 \mu$ and $0.630 \mu$ hue discrimination becomes so poor that $\Delta \lambda$ cannot be measured with precision. A further point of interest is the notch in the curve at $0.570 \mu$

The measurements were made with the colorimeter developed by Dr. W. D. Wright ${ }^{1,2}$. The right eye was used and the field brightnes was approximately 50 photons. A careful check of the wave-length calibration of the colorimeter was undertaken, and to make certain
that the effect was not due to an error within the apparatus a small reversing prism was fltted to the eyepiece so that the position of the upper and lower beams was reversed. Since the lower fleld (now illuminated by the upper beam) still remained slightly more blue than the upper one, the effect could scarcely be due to faulty wave-length calibration.

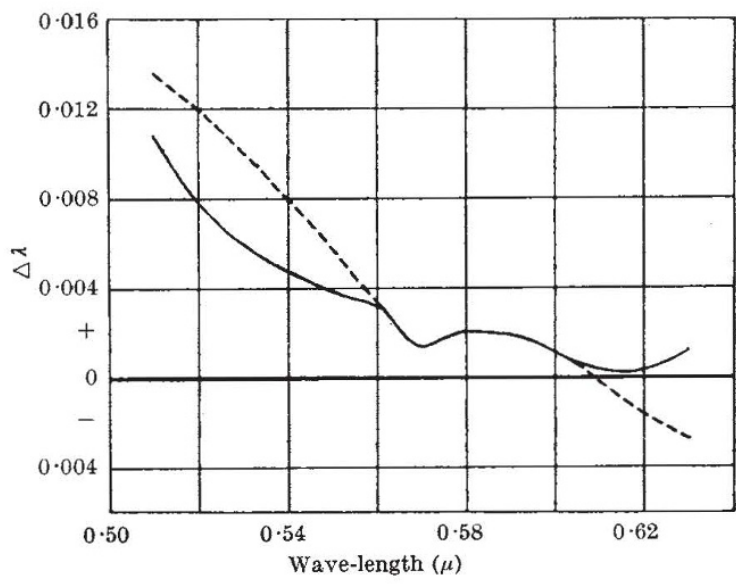

The size of $\Delta \lambda$ (see graph) for any wave-length is only about half that of the hue discrimination step for the same wave-length under the same conditions, and $\Delta \lambda$ is merely the difference of wavelength which makes a colour match unsatisfactory, and is not sufficient for one to be able to record a reproducible hue step. The need for some adjustment of the wave-length cont

ever, was readily seen by the observer.

Thomson and Wright ${ }^{3}$ have shown that there is an increase of sensitivity to blue light as the image of the matching fleld is moved from the centre towards the edge of the central fovea, and the explanation of the thon the colour difference here described upon areas of the point was such that the two halves of the field lay upon areas of the retina of slightly different spectral sensitivity. The irregular shape of the curve may be due to the image of the matching field having a retinal position which depends upon the wave-length. Some diference in the anatomical position of the flxation point for red, green and blue light has been reported by Hartridge ${ }^{4}$ and it may be that the position of the fxation point is a continu

I would like to express my thanks to Dr. W. D. Wright for his help and to Miss M. Gilbert for recording the observations. My thanks are also due to the Medical Research Council for the use of the colorimeter at Imperial College.

Physiology Laboratory,

I. C. Thомson

Guy's Hospital Medical School,

London, S.E.1.

May 10.

${ }^{1}$ Wright, W. D., Trans. Opt. Soc. Lond., 29, 225 (1927).

2 Wright, W. D., J. Sci. Instru., 16, 10 (1939).

3 Thomson, $\dot{\mathrm{L}}$. C., and Wright, W. D., in the press.

- Hartridge, H., Nature, 157, 482 (1946).

\section{Interstellar Origin of Cosmic Radiation at Radio-Frequencies}

RECENT publications of measurements of radiation from interstellar space $^{1}$ and from the sun ${ }^{2,3}$ have important astronomical consequences. In the measurement of cosmic radiations at 5 metres wave-length, the maximum value of $13.2 \times 10^{-21} \Delta v \Delta \omega$ watts/sq. metre was obtained. This is equivalent to a radiation intensity of $4 \times 10^{-18} \mathrm{ergs} /$ sec./kilocycle band-width/sq. cm./sq. degree. We have previously investigated ${ }^{4}$ the theory of interstellar radiation arising from free-free investigated the theory of the fleld of protons. From astronomical observations the numbers of protons and electrons in interstellar space, in the neighbourhood of the sun, are known to be of the order space, in the neighbourhood of the sun, are known to be of the order intensity of radiation at the earth; the maximum predicted value is intensity of radiation at the earth; the maximum predicted value is found to be $5 \times 10^{-18} \mathrm{ergs} / \mathrm{sec} . / \mathrm{kc} . / \mathrm{sq} . \mathrm{cm}$. $/ \mathrm{sq}$. degree, at about 1 metre wave-length. The accompanying graph shows the predicted free-free radiation from interstellar space as well as the presently available high-frequency measures. The plotted observations are the observed maximum intensities, in the region of Sagittarius. Data at the slightly longer wave-length bands, near 10 metres, would be of critical importance. The new and accurate observations by Hey, Phillips, and Parsons lie remarkably close to our predicted curve. Their observations show relatively low concentration of the radio-frequency radiation to the galactic plane, compared to both interstellar matter and stars. explain

this effect. at 1.5 metres, have observed intense short-wave bursts of radiation from the sun, connected with sunspot activity. The energies received attain high values; their data, are given in terms of an "equivalent temperature'. After reduction to our units, the observed intensities are respectively of the order of $5 \times 10^{-13}$ and $3 \times 10^{-14} \mathrm{ergs} / \mathrm{sec} . / \mathrm{kc}$. sq. cm./sq. degree. Pawsey, Payne-Scott and McCready ${ }^{3}$ question the origin of the cosmic radiation in interstellar space, and attribute it to similar [that is, to the sun] bursts of radiation from the stars, which, because of their large number, could yield an approximately constant value for any one area in the sky". We wish to point out some con-
siderations which seem to vitiate this interesting new suggestion. In 